\title{
La théorie et ses abîmes
}

Herbert Spencer dans Le Disciple de Paul Bourget

\section{Niklas Bender}

\section{(CpenEdition}

\section{Journals}

Édition électronique

URL : http://journals.openedition.org/aes/292

DOI : 10.4000/aes.292

ISSN : 2258-093X

Éditeur

Laboratoire LISAA

\section{Référence électronique}

Niklas Bender, "La théorie et ses abîmes », Arts et Savoirs [En ligne], 4 | 2014, mis en ligne le 15 mai

2014, consulté le 06 mai 2019. URL : http://journals.openedition.org/aes/292 ; DOI : 10.4000/aes.292

Ce document a été généré automatiquement le 6 mai 2019.

Centre de recherche LISAA (Littératures SAvoirs et Arts) 


\title{
La théorie et ses abîmes
}

\author{
Herbert Spencer dans Le Disciple de Paul Bourget
}

\author{
Niklas Bender
}

1 Paul Bourget (1852-1935) offre l'exemple d'une réception ambiguë de l'œuvre d'Herbert Spencer en France, réception partielle et partiale, dans laquelle se confondent l'acquiescement et la critique, la fascination et le rejet. L'écrivain français fait au philosophe anglais l'honneur de servir de modèle à un personnage-clé dans Le Disciple $(1889)^{1}$, un roman à thèse; en revanche, l'image quelque peu caricaturale qui y est brossée n'est guère flatteuse. Toutefois, dans son ambiguïté même, elle offre l'occasion d'analyser deux opérations intéressantes: d'abord, la transformation de l'œuvre de Spencer, car ses lecteurs français soulignent de façon excessive certains traits de son œuvre, pour en négliger bien d'autres. Ensuite, l'emploi stratégique des idées de Spencer, car elles servent de pièces à conviction dans les débats intellectuels de la fin du XIX siècle. Sous la plume de Bourget, l'image de Spencer contribue à une critique fondamentale de la philosophie à orientation scientifique, analytique, positive; cette critique est prononcée au nom de la morale. Elle comporte plusieurs volets, que nous tenterons d'expliquer.

2 Le titre du roman l'indique, Le Disciple se construit autour d'une relation de maître à élève; il s'agit bien d'un roman sur ceux qu'on appellera, lors du procès Dreyfus, les «intellectuels $»^{2}$. La relation entre Adrien Sixte ${ }^{3}$, philosophe, et son élève Robert Greslou, jeune précepteur, fournit la constellation de base, une hiérarchie qui sera inversée au cours du roman. Conformément à cette constellation, l'architecture du récit comporte deux niveaux. Le récit cadre, focalisé sur Sixte, relate comment ce dernier se voit mêlé à une affaire d'homicide : en février 1887, il est informé de l'inculpation de Greslou, accusé de l'homicide de Charlotte de Jussat-Randon, la sœur de son élève, morte empoisonnée. L'instruction judiciaire met en cause la responsabilité morale de Sixte, qui, par ses enseignements philosophiques, aurait posé les fondements aux actions de Greslou : dans Le Disciple, les interrogations philosophiques se présentent dans la perspective de leurs conséquences éthiques et sociales. Sixte réfute les accusations, en arguant qu'il n'y a eu que deux entrevues entre lui et Greslou, en août 1885 (81). Néanmoins, le "philosophe moderne » (57) est interrogé puis, la mère de Greslou lui rend visite, afin de le convaincre 
de l'innocence de son fils. Pour appuyer ses propos, elle lui transmet un manuscrit de ce dernier: la lecture du texte convainc Sixte de s'engager en faveur de Greslou - il reconnaît sa propre responsabilité morale. Sixte assiste au déroulement du procès qui se termine par une disculpation. Toutefois, la catastrophe finale est inévitable: le comte André, frère de Charlotte, tue Greslou.

3 À son tour, le récit encadré rapporte le point de vue du disciple: dans sa "Confession d'un jeune homme d'aujourd'hui ", Robert Greslou relate son enfance, sa jeunesse et surtout son année passée chez les Jussat-Randon, qui conduit à la séduction et au suicide de Charlotte. La «Confession » représente plus de la moitié du roman ${ }^{4}$.

Comment Herbert Spencer se trouve-t-il impliqué dans ce récit? Bourget nous l'indique d'emblée : Adrien Sixte est conçu sur le modèle du philosophe anglais, car on le nomme « le Spencer français » (58). Il convient donc d'analyser de plus près le personnage. Adrien Sixte, âgé de 48 ans (61), est présenté comme un moine de l'esprit, il habite Paris comme il pourrait habiter un ermitage (73). Son «existence monastique " (71) est entièrement consacrée au travail, elle tend vers une " complète absorption de l'esprit dans les idées " (61). Sa physionomie en porte les traces, il a le « front haut et fuyant, une bouche avancée et volontaire avec des lèvres minces, un teint bilieux, des yeux malades d'avoir trop lu, [... ] un corps grêle avec de gros os, uniformément vêtu d'une longue redingote " (61 sq.) bref, une physionomie d'intellectuel. Son corps existe uniquement en tant que support négligé d'un esprit hypertrophié. Dans cette description, on discerne difficilement un portrait de Spencer : ce dernier n'a nullement vécu en ermite, sa fréquentation de clubs londoniens et ses voyages sont connus. Surtout, si Bourget avait voulu faire une description caricaturale du philosophe anglais, il aurait probablement souligné le trait caractéristique le plus important de ce dernier, c'est-à-dire sa maladie chronique (qui aurait par ailleurs bien complété l'impression recherchée d'un corps à l'abandon). Physiquement, il ne faut donc nullement voir en Sixte un simple pendant romanesque de Spencer. Ce premier constat vaudra partiellement pour la suite, car, disons-le d'emblée, Sixte est un personnage composite, dans lequel on retrouve maintes traces, parmi lesquelles celle de Spencer nous semble toutefois particulièrement importante, même si, dans le portrait de Sixte, la référence à Emmanuel Kant est également révélatrice. Le train de vie de Sixte est « aussi régulier, aussi maniaque dans ces faits et gestes » que celui du philosophe de Königsberg (57 sq.) : Bourget dessine une image caricaturale du philosophe moderne, dont Kant a fourni un modèle majeur; il représente le penseur dont la vie est entièrement soumise à une réflexion rigoureuse, à tel point qu'elle est réglée comme une horloge. On est d'ailleurs peu surpris d'apprendre que le père de Sixte était horloger (66) - nous reviendrons sur ce détail important. Chez Bourget, la connotation négative d'un tel style de vie est évidente, Sixte est "emprisonné[] [...] dans l'atmosphère des spéculations abstraites » (61), sa physionomie est « comme détruite » par la pensée (ibid.), il a déjà souffert d'une " "violente encéphalite" » faute aux excès intellectuels (67). Nous reviendrons également au portrait critique de Sixte ${ }^{5}$.

De l'homme à l'œuvre, le parallèle avec Spencer se précise dans la présentation de la philosophie de Sixte. D'ailleurs, l'esquisse de tout un système de pensée constitue l'une des réussites du roman. Sixte est un philosophe matérialiste, tourné vers une explication nouvelle de l'esprit humain. Il se fait connaître grâce à son premier livre, la Psychologie de Dieu (68), titre qui, selon Anatole France, serait à traduire par « Étude sur les divers états d'âme dans lesquels l'idée de Dieu a été élaborée " ${ }^{6}$. En effet, 
La thèse de l'auteur consistait à démontrer la production nécessaire de «l'hypothèse-Dieu» par le fonctionnement de quelques lois psychologiques, rattachées elles-mêmes à quelques modifications cérébrales d'un ordre tout physique. Cette thèse était établie, appuyée, développée avec une âpreté d'athéisme qui rappelait les fureurs de Lucrèce contre les croyances de son temps. [...] On n'avait pas rencontré, depuis des années, une pareille puissance d'idées générales mariée à une telle ampleur d'érudition, ni une si riche abondance de points de vue unie à un si audacieux nihilisme. (69 sq.)

Bourget compare cette monographie fictive avec De l'Intelligence d'Hippolyte Taine et La Psychologie anglaise contemporaine de Théodule Ribot (68). Il s'agit d'une analyse psychologique faisant tout reposer sur une réduction physiologique : la vie morale est rapportée au jeu des sensations. Cette réduction s'applique également aux représentations métaphysiques, d'où l'athéisme des propos ${ }^{7}$. Les deux autres ouvrages de Sixte - Anatomie de la volonté et Théorie des passions - ont le même sujet et vont dans la même direction. Bourget résume « la doctrine de M. Sixte » ainsi :

[...] l'auteur [...] admet que l'esprit est impuissant à connaître des causes et des substances, et qu'il doit seulement coordonner des phénomènes. Avec les psychologues anglais, il admet qu'un groupe parmi ces phénomènes, celui qui est étiqueté sous le nom d'âme, peut être l'objet d'une connaissance scientifique, à la condition d'être étudié d'après une méthode scientifique. (73)

$\mathrm{Au}$ premier abord, Sixte défend donc une méthode positiviste, puisqu'il renonce à connaître les causes, et se limite à étudier les phénomènes. C'était déjà la devise d'Auguste Comte dans le Discours sur l'esprit positif (1844) :

En un mot, la révolution fondamentale qui caractérise la virilité de notre intelligence consiste essentiellement à substituer partout, à l'inaccessible détermination des causes proprement dites, la simple recherche des lois, c'est-àdire des relations constantes qui existent entre les phénomènes observés. ${ }^{8}$

Dans son approche générale, Sixte est en accord avec la nébuleuse positiviste; Bourget le souligne, Sixte rejoint les théories de Taine et de Ribot - Spencer lui-même n'est pas loin, puisqu'on peut imaginer qu'il figure parmi les «psychologues anglais » cités dans la foulée ${ }^{9}$.

7 Mais Sixte va plus loin, car il défend deux points de vue qui se distinguent par leur radicalité. Il faut les analyser par le menu, afin de s'en faire une idée précise. Voici les piliers de la doctrine sixtienne :

Le premier réside dans une analyse négative de ce qu'Herbert Spencer appelle l'Inconnaissable. On sait que le grand penseur anglais admet que toute réalité repose sur un arrière-fonds qu'il est impossible de pénétrer ; par suite, il faut [...] comprendre cet arrière-fonds comme incompréhensible. Mais, comme l'atteste fortement le début des Premiers Principes, pour M. Spencer cet Inconnaissable est réel. Il vit, puisque nous vivons de lui. De là il n'y a qu'un pas à concevoir que cet arrière-fonds de toute réalité enveloppe une pensée, puisque notre pensée en sort, un cœur, puisque notre cœur en dérive. Beaucoup d'excellents esprits entrevoient dès aujourd'hui une réconciliation probable de la Science et de la Religion sur ce terrain de l'Inconnaissable. Pour M. Sixte, c'est là une dernière forme de l'illusion métaphysique et qu'il s'est acharné à détruire [...]. (73 sq.)

Le «Spencer français » s'attaque donc à l'auteur des Premiers Principes. Ce dernier texte, paru en 1862, a été traduit en français en 1871 par Émile Cazelles, et il a impressionné Bourget lors de sa lecture vers $1878^{10}$. Spencer y établit effectivement le principe de l'inconnaissable : «Si la religion et la science peuvent se réconcilier, c'est sur ce fait, le plus profond, le plus large et le plus certain de tous : que la puissance dont l'univers est la manifestation pour nous est complètement impénétrable. ${ }^{11}$ Spencer fait cette 
observation afin de définir le domaine du connaissable, appartenant à la Science; il l'oppose à l'inconnaissable, domaine de la Religion. Cette délimitation des domaines et des fonctions a pour finalité une "réconciliation », dans le cadre d'un progrès général de l'esprit humain :

Le progrès de l'intelligence a toujours été double. Chaque pas en avant a rapproché à la fois du naturel et du surnaturel [...]. À mesure que la science s'élève vers son apogée, tous les faits inexplicables et en apparence surnaturels rentrent dans la catégorie des faits explicables et naturels. En même temps, on acquiert la certitude que tous les faits explicables sont à leur origine première inexplicables et surnaturels. De la sorte naissent deux états antithétiques de l'esprit répondant à des côtés opposés de cette existence qui fait l'objet de notre pensée. (PP 93)

Ainsi, la Science et la Religion représentent « deux modes antithétiques de la conscience » (PP 94) qui sont également complémentaires. Si Spencer en vient à parler d'une « cause inconnue » (PP 108 sq.), il admet lui-même que le principe ainsi établi est peu satisfaisant du point de vue théologique: «L'immense majorité des hommes rejettera avec indignation une croyance qui paraît si impalpable et si mal arrêtée.» (PP 100) Contrairement à ce que suggère la critique énoncée par Sixte, Spencer n'entend pas établir une causalité métaphysique. Son entreprise est elle-même critique, dans la tradition kantienne $:$ il analyse les limites de la connaissance humaine. On peut en voir un indice révélateur dans le fait que Spencer écrit « inconnaissable » en minuscule ${ }^{12}$.

Or, Bourget prétend que Spencer défend une notion de l'Inconnaissable qui serait d'abord du domaine psychologique. Ceci n'est pas tout à fait faux, Spencer ayant lui-même retrouvé le principe de l'inconnaissable dans la vie psychique; ceci lui permet de tenir à l'écart à la fois les matérialistes et les spiritualistes ${ }^{13}$. Bourget va plus loin en disant que cet inconnu aurait un fondement métaphysique ${ }^{14}$, et c'est à cette notion que Bourget laisse Sixte s'attaquer - dans la perspective d'inverser l'argument, puisque le roman donnera tort à Sixte.

La référence à Spencer est donc bien complexe: Bourget présente un Sixte qu'il apparente d'abord superficiellement à Spencer. Cependant, il s'avère rapidement qu'au fond, Sixte défend des positions contraires à la philosophie spencérienne; la ressemblance se limitant à un certain type de renommée intellectuelle. En même temps, de façon plus fondamentale, Bourget oppose Sixte à Spencer, afin de donner raison à ce dernier - mais à un Spencer qu'il a façonné de ses mains ${ }^{15}$. Spencer apparaît donc doublement dans Le Disciple, et chacune des représentations modifie fortement son modèle.

Pour l'instant, venons-en au second point caractéristique de la philosophie de Sixte :

Son second titre d'honneur, comme psychologue, consiste dans un exposé très nouveau et très ingénieux des origines animales de la sensibilité humaine. Grâce à une lecture immense et à une connaissance minutieuse des sciences naturelles, il a pu tenter pour la genèse des formes de la pensée le travail que Darwin a essayé pour la genèse des formes de la vie. Appliquant la loi de l'évolution aux divers faits qui constituent le cœur humain, il a prétendu montrer que nos plus raffinées sensations, nos délicatesses morales les plus subtiles, comme nos plus honteuses déchéances, sont l'aboutissement dernier, la métamorphose suprême d'instincts très simples, transformation eux-mêmes des propriétés de la cellule primitive; en sorte que l'univers moral reproduit exactement l'univers physique et que le premier n'est que la conscience douloureuse ou extatique du second. (74 sq., je souligne)

En fait, le narrateur attribue deux innovations distinctes à Sixte : d'un côté, l'application de la loi de l'évolution au domaine de la psychologie ${ }^{16}$, de l'autre, la réduction de la vie 
psychique à la vie physiologique. La première innovation renvoie à Spencer dans la mesure où ce dernier est bien le psychologue qui applique la théorie de l'évolution au domaine de l'âme. Cependant, il ne s'agit justement pas de la loi de l'évolution de Darwin, mais d'une loi bien plus générale, appartenant davantage au domaine de la philosophie qu'à celui de la science naturelle proprement dite :

L'évolution est une intégration de matière accompagnée d'une dissipation de mouvement, pendant laquelle la matière passe d'une homogénéité indéfinie, incohérente, à une hétérogénéité définie, cohérente, et pendant laquelle aussi le mouvement retenu subit une transformation analogue. (PP 355)

Cherchant à établir une loi universelle, Spencer a recours à ce concept spéculatif d'évolution, applicable à tous les domaines, de l'astronomie à la zoologie, de la géologie à la sociologie, ce que montre très bien le chapitre XIV des Premiers principes, «La loi de l'évolution".

Quant à la seconde innovation de Sixte : si Spencer propose une continuité entre l'esprit et la physiologie, il ne tente pas de réduire le premier à la seconde contrairement à la conception de Sixte. La loi de l'évolution spencérienne implique l'émergence progressive de sentiments plus complexes ${ }^{17}$ et une adaptation, une recherche d'équilibre entre le monde intérieur et le milieu extérieur. On peut tout à fait avancer qu'en dernière conséquence, sa philosophie de l'évolution aboutit à une réduction du psychique au physique - mais ce n'est guère la volonté principale de Spencer ${ }^{18}$.

Résumons : sur l'échiquier de la philosophie contemporaine, Bourget place Sixte dans une position extrême. Sixte s'éloigne du positivisme, car il nie le libre arbitre et il défend «le déterminisme le plus complet» (75). Bref, il ose la synthèse dans le sens d'un matérialisme radical, il tranche là où le positivisme se refusait à trancher. Qui plus est, dans le domaine de la morale, il en résulte un relativisme total. Les valeurs morales constituent "un ensemble de conventions quelquefois utiles, quelquefois puériles", affirme-t-il devant le juge d'instruction: «[...] pour le philosophe il n'y a ni crime ni vertu. Nos volitions sont des faits d'un certain ordre régis par certaines lois, voilà tout. » (97) Voici le cœur du problème, exposé par un procédé d'exagération qui frôle parfois la caricature.

Sixte ne tarde pas à être critiqué pour ses prises de position : la voix du narrateur émet des réserves, mais c'est surtout l'histoire de Greslou qui sert de pierre de touche. Par surcroît, le disciple devient un critique sévère du maître : dans sa "Confession ", il relate les événements passés en les jugeant du haut de son expérience acquise. La critique de Sixte comporte donc deux faces: elle est implicitement contenue dans le résultat de l'application, et elle est explicitement énoncée dans les conclusions de Greslou, porteparole de Bourget.

Cependant, toute la complexité du roman est à voir dans la façon dont cette critique de Sixte est liée à l'implication de Spencer. Cette dernière est patente sur au moins trois plans, qui représentent autant de facettes de la critique de Sixte : celui de la démarche générale de Greslou; celui de ses motivations dans son entreprise de séduction; celui, enfin, de l'application concrète de l'enseignement philosophique. Examinons-les successivement.

16 La première critique touche à la démarche générale de Greslou. Ce jeune homme intelligent emploie la méthode psychologique de son maitre en analysant deux sujets, luimême et Charlotte. Le lecteur n'apprend pas tout des procédés exacts, car le journal de l'expérience se perd en route; il n'empêche que les grandes lignes se dessinent au cours 
du récit. Aux yeux de Greslou, la démarche psychologique se présente comme toute naturelle. Le précepteur a, depuis la plus tendre enfance, une "faculté de dédoublement " $(190)^{19}$ qui lui vient d'un manque d'identification avec son milieu, et surtout avec sa mère, esprit simple et d'une moralité pieuse. Cette faculté de dédoublement ${ }^{20}$, réunissant l'action à l'observation de soi, est douteuse d'un point de vue moral, car elle implique un jeu de masques, et donc la fausseté. Ainsi, la démarche scientifique, proposée par Sixte en toute bonne foi, renforce un mauvais trait de caractère chez son acolyte - ce schéma revient à plusieurs reprises. La préférence nette de l'analyse sur l'action contribue par exemple à développer unilatéralement l'esprit de Greslou: "Sous l'influence de vos livres, mon cher maître, et sous celle de votre exemple, je m'étais intellectualisé de plus en plus.» (188) Par son penchant naturel et par les enseignements de Sixte, Greslou devient « une froide et meurtrière machine à calcul mental » (192). Dans cette démarche de Sixte, on peut - de façon tout à fait générale - reconnaître les tentatives d'observation et d'explication psychologique qui caractérisent l'œuvre de Spencer, mais également celle des penseurs français travaillant dans son sillage.

De la démarche du disciple, on peut déduire une critique sévère du maître: par ses enseignements, Sixte pousse à une existence purement intellectuelle, il forme un homme « incomplet » (189), séparé de l'action et de l'amour véritable. Au fond, l'esprit d'analyse psychologique se passe de toute attache extérieure, du corps, d'autrui, et se pose en maître absolu - de là la tendance de Greslou à développer un culte du Moi. Par conséquent, il est également dénué de critères éthiques, alors qu'il touche au domaine de la vie morale; les conséquences seront désastreuses.

La deuxième critique porte sur les motivations de Greslou lors de la séduction de Charlotte. Greslou croit agir par esprit scientifique, il voudrait mener une expérience sur le cœur humain. En réalité, il agit par désir, sentiment auquel se mêle l'orgueil du parvenu. Tel Julien Sorel, il est fier de séduire une femme de noble extraction (205). La haine, enfin, est un autre moteur puissant : Greslou admire et déteste à la fois le comte André, le frère de Charlotte. Face à ce militaire, homme d'action et d'honneur, Greslou ressent l'impuissance de l'homme cérébral et de basse extraction. Dans son esprit, une « rivalité » s'installe (181), et Charlotte devient le «champ de bataille pour la secrète, pour l'obscure antipathie » qu'il ressent envers le comte (196).

Greslou formule lui-même cette interprétation, dans sa confession tardive ; il s'agit d'une explication après-coup. En fait, Greslou restera un mystère à lui-même, avec l'évolution notable qu'au moment des faits, il n'en est pas conscient, alors qu'il le devient plus tard. $\mathrm{Au}$ moment de sa confession, il voit clairement son «inintelligence » d'alors, il constate que toute son entreprise de séduction était une « comédie à demi instinctive » (201). Tel un Valmont scientifique, il croyait agir sur Charlotte, alors qu'il suivait ses propres désirs obscurs. Son comportement prouve également, ce qui est tout à fait dans l'esprit de Spencer, que la Mémoire, la Raison et les Sentiments n'existent pas séparément, mais qu'ils se manifestent toujours conjointement ${ }^{21}$. La finalité de l'argumentation diffère pourtant : par l'exemple de Greslou, Bourget suggère que le scientifique en général n'est pas conscient des causes qui motivent son action.

À propos de l'objet de ses études, Greslou n'est pas plus pertinent. Loin d'être une victime manipulée par un expérimentateur habile, Charlotte suit simplement les lois de la Nature :

Il se jouait tout entier en elle et sans que j'y comprisse rien, ce drame où la Mort et l'Amour, les deux fidèles ouvriers de l'implacable Nature, ont agi sans mon ordre et 
en se moquant des complications de mes analyses. Charlotte m'a aimé pour des raisons absolument différentes de celles qu'avait su aménager ma naïve psychologie. (201sq.)

Cette autocritique de "[l']écolier dans la science du cœur» (201), variée maintes fois au cours du récit, permet d'élargir la critique de Sixte - elle devient fondamentale. Par la voix de Greslou, Bourget fait valoir que l'homme est un mystère, tant à lui-même qu'aux autres. L'inconnaissable spencérien est ici détourné et repris afin d'attaquer les psychologues matérialistes et déterministes : en croyant que l'esprit humain se comprend et s'explique totalement, Sixte commet à la fois une erreur intellectuelle et une faute morale.

$\mathrm{Au}$ fond, Bourget pointe le problème des modèles de causalité. Greslou, en disciple de Sixte, se voyait «suivre dans [1] es yeux [de Charlotte] les mouvements qui l'agitaient comme on suit les allées et venues du mécanisme d'une montre à travers une boîte en cristal» (200). C'est l'analogie qui fait de l'esprit une horloge ${ }^{22}$, le psychologue étant l'horloger ; il faut rappeler ici que le père de Sixte était horloger et que l'emploi du temps $\mathrm{du}$ philosophe reproduit une exactitude conforme à la profession paternelle ${ }^{23}$. Or, l'analogie entre l'homme et l'horloge est erronée :

Pour définir les phénomènes du cœur, c'est au monde végétal qu'il faut emprunter des analogies et non à la mécanique. Pour conduire ces phénomènes, c'est des procédés de botaniste qu'il convient d'employer [...]. Un sentiment naît, grandit, s'épanouit, se dessèche comme une plante, par une évolution parfois ralentie, parfois rapide, toujours inconsciente. (212, je souligne)

Selon cette deuxième image, l'esprit se compare à une plante ; ses racines renvoient à son versant obscur, inconscient, inaccessible. Son développement ${ }^{24}$ serait pareil à une évolution, terme dont le double sens renvoie bien à Spencer. Mais le processus visé n'est justement pas une loi limpide et universelle, illustrant le progrès. Tout au contraire, Greslou/Bourget évoquent ici le concept de l'évolution pour désigner la part opaque, incompréhensible de l'homme. Une nouvelle fois, Spencer est convoqué pour critiquer les conceptions de Sixte, mais dans un sens qui diffère des intentions du philosophe anglais.

Finalement, l'application concrète des enseignements psychologiques révèle leur caractère problématique. Greslou tente de séduire Charlotte en manipulant ses sentiments ; dans sa démarche, il se base sur la Théorie des passions de Sixte. Prenons deux exemples. Le premier se trouve dans l'emploi de la pitié : Greslou détourne une vertu chrétienne, procédé particulièrement cynique. À ce propos, il cite la Théorie des passions : « Il y a dans ce phénomène de la pitié un élément physique et qui, chez les femmes particulièrement, confine à l'émotion sexuelle..." (203) Cette observation d'ordre théorique sera transformée en précepte pratique: Greslou invente une histoire d'amour déçu qui ne manquera pas de produire son effet sur Charlotte. Ainsi, en dernière conséquence, la pitié mènera effectivement à l'acte sexuel.

Une deuxième observation légitimera ce passage à l'acte :

Je prenais votre Théorie des Passions, et j'y étudiais vos phrases sur le duel des sexes dans l'amour. - «C'est la loi du monde », raisonnais-je, « que toute existence soit une conquête, exécutée et maintenue par le plus fort aux dépens du plus faible. Cela est vrai de l'univers moral comme de l'univers physique. Il y a des âmes de proie comme il y a des loups, des chats-pards et des éperviers. » (245)

Greslou se présente en prédateur, Charlotte étant sa proie : c'est un darwinisme appliqué à l'amour qui sert de justification. Concernant l'égoïsme de son personnage, Bourget ne cite pas Spencer; or, cela aurait été une rare occasion pour le faire. Selon Spencer, 
l'égoïsme est la loi générale de la vie sociale et un moteur nécessaire à son développement ; il n'admet l'altruisme que par calcul ${ }^{25}$.

Dans les deux cas d'application pratique, Greslou isole d'abord des observations dans les réflexions théoriques de Sixte; ces réflexions sont fondées sur une conception matérialiste et déterministe de la psychologie humaine. Greslou transforme ce déterminisme psychologique en mode d'emploi : il utilise la pitié dans son intérêt et justifie sa démarche par le recours au darwinisme. Du point de vue de Bourget, le test pratique sert à démasquer le cynisme inhérent à la théorie ${ }^{26}$. Son but est d'apporter une preuve a contrario : en montrant les conséquences pratiques et éthiques de la psychologie moderne, il suggère le retour aux valeurs morales ${ }^{27}$, à la religion chrétienne. Voici la leçon finale du roman : ébranlé par les agissements de son disciple, face à la catastrophe finale, Sixte se souvient: «[...] cet analyste presque inhumain à force de logique s'humiliait, s'inclinait, s'abîmait devant le mystère impénétrable de la destinée. Les mots de la seule oraison qu'il se rappelât de sa lointaine enfance: "Notre Père qui êtes aux cieux..." lui revenaient au cœur. » (327)

$\mathrm{Au}$ total, les chefs d'accusation sont donc les suivants : premièrement, le psychologue libère l'intellect de toutes les attaches; il le tient pour absolu, tout en négligeant et le corps et la morale. Deuxièmement, il se trompe sur la nature de cet intellect, qui n'est pas limpide : il garde toujours une part obscure. Troisièmement, son analyse rabaisse l'esprit humain et ouvre par là les portes au cynisme; l'orgueil aveugle de l'esprit et son abaissement matérialiste vont main dans la main.

Mais quel philosophe occupe ici le banc des accusés ? Hugh M. Davidson ${ }^{28}$, Dirk Hoeges et Sophie Spandonis suggèrent que Spencer/Sixte sert ici de nom de code pour Taine ${ }^{29}$. Cela est certainement vrai concernant quelques critiques précises proposées par le truchement du personnage, comme celle de l'inconnaissable spencérien. Pourtant, il semble plus pertinent à notre avis de voir Sixte comme une incarnation de l'intellectuel type de la Troisième République, qui se base sur plusieurs modèles, dont celui bien plus ancien de $\mathrm{Kant}^{30}$.

La présence de Spencer chez Bourget pose donc un problème de degré : dans quelle mesure ses réflexions sont-elles reprises dans Le Disciple? D'un côté, elles figurent de toute évidence parmi les positions critiquées. Tout comme Spencer, Sixte privilégie une démarche globalement positiviste. Tout comme Spencer, il explique la genèse des sentiments comme évolution. Dans le débat sur la place de la Science au sein de la société, qui fait rage à la fin du XIX ${ }^{\mathrm{e}}$ siècle et qui se poursuit dans Le Disciple, Bourget attaque Spencer (ou l'attaque potentiellement) pour les prises de position qui le relient au positivisme et au scientisme.

D’un autre côté, Spencer est justement évoqué dans le but de critiquer Sixte. La notion spencérienne d'inconnaissable est appliquée à la vie psychique, pour démontrer sa nature insondable. L'emploi de la notion d'évolution va dans le même sens. À y regarder de près, Bourget évoque un Spencer bien particulier. Chez Spencer, le domaine de l'inconnaissable a pour fonction de tenir à équidistance le spiritualisme et le matérialisme. Bourget, en revanche, évoque l'Inconnaissable pour lui accorder une existence à part, voire pour en faire une entité métaphysique - bref, il l'emploie pour attaquer le positivisme et pour verser dans la théologie; Taine ne s'y trompe pas ${ }^{31}$. Le même constat vaut pour l'évolution : pour Spencer, il s'agit d'une loi de progrès, alors que pour Bourget, elle sert à renvoyer à la part d'ombre de l'homme. Bourget emploie la philosophie de Spencer pour 
établir une psychologie de l'Inconscient à fondement métaphysique. Il façonne sa référence: la démarche encyclopédique de Spencer - comprenant l'épistémologie, la biologie, la psychologie, la sociologie, l'éthique, pour ne nommer que les parties de son système que Spencer a pu réaliser pleinement - est rétrécie à la vie psychique, et au domaine de la Foi.

La tentative de récupération cache surtout une différence fondamentale : la philosophie de Spencer est marquée par l'optimisme caractérisant le milieu du XIX siècle, il croit pouvoir réconcilier Progrès, Évolution, et Foi ${ }^{32}$. Pour lui, au fond, tous les domaines abordés ne font qu'un, même si le lecteur d'aujourd'hui croit y déceler un biologisme et un social darwinisme larvés. Pour Bourget, en revanche, un fossé se creuse entre corps et esprit, entre réflexion et action, entre Science et Religion. Sa finalité est de mettre en garde la jeunesse, dans le souci de sa vitalité physique et morale. Sa préface «À un jeune homme » en fournit l'illustration.

Pourtant, malgré son acharnement, la critique de la philosophie scientifique est ambiguë. Dans Le Disciple, certains éléments prennent un contre-pied, soit direct, soit implicite. Car la critique de la Science ne se fait pas au nom d'un irrationalisme pur et simple, le roman à thèse préfère l'argumentation, et la preuve par l'expérience. En quelque sorte, il ressemble à André Cornélis (1887) en ceci qu'il est également « un roman d'analyse exécuté avec les données actuelles de la science de l'esprit $»^{33}$. Il accepte les armes de l'adversaire, et leur confère ainsi une certaine légitimité. Le narrateur critique même le philosophe au nom de l'empirisme, car Sixte ne se pose pas la question si Greslou est vraiment un meurtrier: "Le philosophe n'y songea même point, s'abandonnant sans s'en rendre compte à ce défaut des esprits généralisateurs qui ne vérifient jamais qu'à demi les données sur lesquelles ils spéculent. » (106) On peut observer un procédé analogue dans la démarche globale de Greslou, qui se reproche ses raisonnements d'alors (192) - tout en continuant à raisonner, pour tirer les leçons de ses expériences. Le respect des faits et la rigueur logique restent les pierres de touche du roman.

Par ailleurs, le roman adopte une structure "scientifique", notamment dans la partie relatée par Greslou: si son titre renvoie à Musset et à la littérature romantique, la "Confession» ressemble néanmoins à un texte scientifique de l'époque ${ }^{34}$. Le récit se divise en paragraphes, intitulés "Mes hérédités " (125-148), «Mon milieu d'idées » (148-168), «Transplantation» (168-191), etc.; le tout semble présenter un cas psychiatrique. Or, la «Confession » représente la majeure partie du roman.

Finalement, de façon générale, le sujet du roman obéit aux préceptes scientifiques tels que Taine les a présentés dans De l'Intelligence ${ }^{35}$. Taine y précise que « la conscience, qui est notre principal instrument, ne suffit pas à l'état ordinaire $»^{36}$, et il recourt donc à « un éclairage plus vif »: les différentes pathologies sont censées aider à comprendre le fonctionnement normal de notre esprit. Tout le vocabulaire du traité s'en trouve modifié, les perceptions sont rebaptisées "illusions", etc. De façon comparable, Le Disciple présente un cas pathologique, marqué par une pluralité du Moi dans l'esprit de Taine ${ }^{37}$. Cet exemple a contrario sert à concevoir un ethos normal, souhaitable; le comportement du comte André en est l'esquisse. On peut donc conclure que Bourget, par les moyens et la structure même de sa critique, affirme au moins partiellement la philosophie scientifique à laquelle il prétend s'attaquer.

Toutes proportions gardées, on peut voir dans Le Disciple une tendance inverse à l'œuvre de Spencer. Au moment du triomphe du positivisme, le philosophe anglais conçoit un 
système philosophique à inspiration scientifique, qui laisse une porte ouverte à la Religion. Au moment de la crise de ce même positivisme, Bourget parle de l'autre côté de cette même porte, car il semble nier la Science au nom de la Religion. En vérité, sa critique s'articule à l'aide de la Science, et à l'aide de Spencer, qui lui fournit la clé (" l'inconnaissable »). Mais il continue à tirer la matière et la forme de son récit de cette même pensée scientifique qu'il soumet à rude épreuve: Bourget aussi refuse les portes fermées, même s'il se place de l'autre côté du seuil.

\section{NOTES}

1. Paul Bourget, Le Disciple, éd. Antoine Compagnon, Paris, Librairie générale française, 2010, coll. «Le livre de poche. Classiques»; par la suite, j'indiquerai simplement la page de cette édition. Pour le contexte de la publication et pour la réception du roman, $c f$. Lloyd-James Austin, Paul Bourget. Sa vie et son œuvre jusqu'en 1889, Paris, Droz, 1940, p. 225-236.

2. Sur l'emploi de ce terme lors de l'affaire Dreyfus, $c f$. Michel Winock, Le Siècle des intellectuels, Seuil, 1999, p. 29-31, coll. « Points »; Winock analyse également le rôle de Bourget (ibid., p. 59, et surtout p. 64 sq.).

3. Le personnage n'est pas neuf dans l'œuvre de Bourget: un philosophe du même nom figure dans le récit cadre de L'Irréparable (étude de jeune fille), de 1884. Mais celui-ci est décrit comme «métaphysicien ", il met en avant " "notre vie inconsciente" "- bref, il défend des positions philosophiques qui se trouvent à l'opposé de celles énoncées par le Sixte du Disciple. Cf. Paul Bourget, L'Irréparable. Deuxième amour. Céline Lacoste. Jean Maquenem, Paris, Plon, 1901 (éd. définitive), p. 3-6, ici p. 3 sq.

4. Son début est inspiré de la jeunesse de Bourget à Clermont-Ferrand (cf. Le Disciple, p. 126, note 1) ; ce caractère autobiographique renforce l'implication de l'auteur. L'histoire de Greslou a un fondement réel, l'affaire Chambige, qui date de 1888 (cf. Compagnon, «Introduction », Le Disciple, op. cit., p. 18-24). Cette affaire, au cours de laquelle Henri Chambige avait tué sa maîtresse, Mme Grille, sans réussir son propre suicide, émeut les esprits des contemporains, e.g. celui de Maurice Barrès ; cf. Emilien Carassus "De l'Affaire Chambige au Jardin de Bérénice », Littératures, $\mathrm{n}^{\circ} 14$, 1991, p.115-125; pour une brève présentation de l'affaire, Marc Renneville, «L'affaire Chambige ", http://m.renneville.free.fr/?p=148 (consulté le $1^{\text {er }}$ août 2013). On peut penser à d'autres affaires judiciaires de l'époque, e.g. le cas Lebiez-Barré (1878) ; cf. Linda L. Clark, Social Darwinism in France, Alabama, University of Alabama Press, 1984, p. 47-50, 110 ; et Albert Autin, Le Disciple de Paul Bourget, Paris, Société française d'éditions littéraires et techniques, 1930, p. 47-53, coll. « Les grands événements littéraires ».

5. Un autre modèle important est Hippolyte Taine : il fournit certaines habitudes à Sixte, comme celle de se promener dans le Jardin des Plantes. Sur la question s'il faut voir dans Taine le modèle principal du personnage, $c f$. la fin de cet article.

6. Chronique dans Le Temps du 23 juin 1889, p. 2 ; Anatole France évoque «Spinosa » [sic] comme modèle de Sixte.

7. La déduction anthropologique et physiologique de la croyance a son origine moderne chez Ludwig Feuerbach, qui inspirera Karl Marx. Cf. Ludwig Feuerbach, Grundsätze der Philosophie der Zukunft (1843), éd. Gerhart Schmidt, tiré de la Kritische Ausgabe, Francfort-sur-le-Main, Vittorio Klostermann, ${ }^{3} 1983$, p. 339 et 343. 
8. Auguste Comte, Discours sur l'esprit positif, éd. Annie Petit, Librairie philosophique, Paris, J. Vrin, 1995 [1844], p. 66. Soit souligné en passant que la recherche d'un système pousse Comte luimême à quitter cette approche, la pensée positive, pour le positivisme proprement dit.

9. Même si Spencer lui-même cherche justement à dépasser le clivage entre Idéalisme et Positivisme, comme le souligne à juste titre David C.J. Lee, «Bourget's Debt to Herbert Spencer : Le Disciple and the Self-Adjusting Watch », Modern Language Review, $\mathrm{n}^{\circ}$ 95, 3, 2000, p. 653-673, ici p. 657. Cependant, Spencer s'inspire surtout du courant positiviste. En France, il est perçu comme une correction de ce dernier, mais venant de l'intérieur. Citons à titre d'exemple la présentation que fait son admirateur Auguste Laugel de lui dans un article connu dans la Revue des Deux Mondes (15 février 1864, p. 930-957, ici p. 934), qui suscite l'ire de Littré ; cf. D.G. Charlton, Positivist Thought in France during the Second Empire 1852-1870, Oxford, The Clarendon Press, 1959, p. 60 sq.

10. Il le dit dans une lettre à Charles Ritter du 13 mai 1902 ; cf. Eugène Ritter, Charles Ritter, ses amis et ses maîtres. Choix de lettres, 1859-1905, Lausanne, Payot, 1911, p. 289 ; et Michel Mansuy, Un Moderne. Paul Bourget. De l'enfance au Disciple, Paris, Les Belles Lettres, 1960, n³8, p. 306, coll. «Annales Littéraires de l'Université de Besançon ». D'autres connaissances sont probables, car Bourget a vraisemblablement lu La Psychologie anglaise contemporaine, École expérimentale de Ribot, publiée en 1870, chez Ladrange ( $c f$. Mansuy, Un Moderne. Paul Bourget, de l'enfance au Disciple, op. cit., p. 151, note 135, et p. 153 sq., note 141).

11. Herbert Spencer, Premiers Principes, trad. Émile Cazelles, Paris, Alcan, 1894, p. 40 ; par la suite, cité par PP.

12. La suite de ses réflexions philosophiques confirme cette observation, car Spencer développe une théorie générale de l'évolution, d'inspiration lamarckienne. Par conséquent, l'esprit humain sera expliqué par les mêmes lois que la biologie, et le fonctionnement de l'intelligence par son adaptation au milieu.

13. Herbert Spencer, Principes de psychologie (1870-1872), Paris, Alcan, 1892, t. I, p. 484 sq., trad. Théodule Ribot et Alfred Espinas (de la nouv. éd.).

14. Cette tendance se trouve déjà dans l'essai «Science et Poésie » (1883), Paul Bourget, CEuvres complètes, Paris, Plon, 1900, t. II : Critique II : Études et portraits, p. 145-174. Deux jeunes gens y débattent de l'avenir de la poésie; leurs différences s'articulent autour de la notion de l'inconnaissable. Le représentant de la Science en minimise la portée: «Entre ce que nous connaissons d'une connaissance scientifique et de l'Inconnaissable, il y a une différence de degré, il n'y a pas une différence d'essence. » (p. 157) Le représentant de la Poésie, en revanche, défend l'idée que "l'Inconnaissable» serait "le Mystérieux", et surtout celui de l'émotion (p. 168). Selon lui, la poésie a pour tâche d'articuler «l'inexprimable comme inexprimable », elle a donc pour sujet un "sentiment tout voisin du mysticisme» (p. 170); il insiste sur «cet insondable cœur humain » comme source du sentiment (ibid.). Tout en privilégiant le jeune poète, Bourget ne tranche pas entre les positions. Toujours est-il qu'il transforme l'inconnaissable en quelque chose de manifeste, lié à la vie psychique; il lui confère un pouvoir nouveau. On le devine : de façon générale, l'inconnaissable de Spencer permet à Bourget de revenir progressivement vers la Foi. Pendant quelque temps, quatre courants de pensée coexistent chez lui: panthéisme, positivisme, religion de l'Inconnaissable et pessimisme ; $c$. Mansuy, Un Moderne. Paul Bourget, de l'enfance au Disciple, op. cit., p. 308 sq. Plus tard, la mission accomplie, Bourget revendiquera un «Inconnaissable » qui ne peut être saisi que «métaphysiquement », et il critiquera les Premier Principes comme "pseudo-scientifiques"; cf. «De la vraie méthode scientifique» (1905), Paul Bourget, Études et Portraits, Paris, Plon, 1906, t. III : Sociologie et Littérature, p. 3-22, ici p. 19 et p. 8. ; cf. également Austin, Paul Bourget. Sa vie et son œuvre jusqu'en 1889, op. cit., p. 210.

15. Pour l'instant, il suffit de constater que Sixte est de nouveau en accord avec Taine, qui a justement critiqué Spencer : «Laissons de côté dans Herbert Spencer la partie faible et arriérée, c'est-à-dire l'hypothèse scolastique d'une substance inconnaissable [...].» Il s'agit d'un article publié dans le Journal des Débats le 4 mars 1874, repris dans Hippolyte Taine, «Études de 
psychologie II : Th. Ribot, Bain, Herbert Spencer ", Derniers Essais de critique et d'histoire, Paris, Hachette, 1923 [1894] (sixième édition), p. 194-205, ici p. 199. Cf. aussi la lettre de Taine à Ribot, du 11 janvier 1873 : Taine y énumère les ressemblances de la nouvelle édition des Principles of Psychology avec De l'Intelligence (en soupçonnant Spencer de l'avoir copié). Mais il retient également les différences : «Par exemple, je diffère absolument de Spencer sur le fond des choses, je ne crois pas du tout qu'il soit inconnaissable ; surtout, je n'admets point que le fond de l'esprit soit inconnaissable. - J'aboutis à croire que les seules réalités de la nature sont, non pas des puissances comme il le dit, mais des événements, sensations et mouvements, les puissances n'étant que la possibilité ou la nécessité de ces événements. » Hippolyte Taine, Sa Vie et sa Correspondance, Paris, Hachette, 1905 [1904], t. III : L'Historien (1870-1875), p. 215-218, ici p. 217.

16. C'est ici une caractéristique de la théorie de Spencer, et non de celle de Taine.

17. Cf. Spencer, Principes de psychologie, op. cit., p. 484 sq. C'est selon Théodule Ribot son exploit le plus important : «M. Herbert Spencer », La Psychologie anglaise contemporaine, Paris, L'Harmattan, 2002 [1870], p. 145-220, ici p. 215 sq.

18. Taine offre dans De l'Intelligence une analyse de l'esprit en fonction de la physiologie: de nouveau, Sixte semble autant suivre le modèle du philosophe français que celui de son homologue anglais. Mais même Taine est moins radical dans son déterminisme que Sixte. Hugh M. Davidson constate ainsi à propos du personnage : " The evidence leads one to think that he is less liberal than is Taine. "Cf. "The Essais de Psychologie Contemporaine and the Character of Adrien Sixte », Modern Philology, n 46, 1, 1948, p. 34-48, ici p. 46.

19. Le manuscrit qu'il soumet à Sixte lors de leur première entrevue porte le titre Contribution à l'étude de la multiplicité du Moi ; Bourget, Le Disciple, op. cit., p. 79.

20. Ici, la référence aux héros de Stendhal est éminente. Anatole France souligne dans sa chronique que Greslou représente un « nouveau Julien Sorel », op. cit.

21. Cf. Spencer, Principes de psychologie, op. cit., p. 509 sq. Ribot souligne ce point, «M. Herbert Spencer ", op. cit., p. 176.

22. Cf. également Bourget, Le Disciple, op. cit., p. 203. Dans cet emploi de l'image de l'horloge, tout le différend entre Bourget et Taine est concentré. Car Bourget s'est certainement inspiré de la «Préface » de De l'Intelligence: «En général, tout état singulier de l'intelligence doit être le sujet d'une monographie; car il faut voir l'horloge dérangée pour distinguer les contrepoids et les rouages que nous ne remarquons pas dans l'horloge qui va bien.» Hippolyte Taine, De l'Intelligence, Paris, Hachette, 1926 [1870] (seizième édition), «Préface », p. 1-22, ici p. 17. Taine s'en défend indirectement : dans une lettre à Bourget du 29 septembre 1889, il critique Le Disciple. Il relève notamment deux points faibles : l'indulgence possible pour Greslou (qui serait « contre la morale ») et la défense des «convictions morales bien arrêtées » que certains pourront tirer du récit. Cette dernière critique est illustrée par l'image de l'horloge : « Ils se sentiront pris [...] dans l'engrenage de votre horlogerie psychologique, mais ce qu'ils éprouveront, quand ils seront tirés par le jeu des rouages, sera de la répugnance, et non de la complaisance, et, enfin, quand ils verront le grand ressort central de tout le mécanisme, je veux dire la théorie des lois naturelles et le déterminisme, ils s'y aheurteront, ils voudront le briser. [...] leur conclusion sera contre la science. " Taine voit la racine du problème dans le caractère de Sixte, qui, à son goût, manque totalement d'expérience - et il renvoie Bourget à Spencer. Taine, Sa Vie et sa Correspondance, op. cit., t. IV : L'Historien (suite). Les dernières années (1876-1893), p. 287-293, ici p. 289 sq. et p. 291. Sur l'image de l'horloge dans Le Disciple, cf. Lee, « Bourget's Debt to Herbert Spencer : Le Disciple and the Self-Adjusting Watch », op. cit., passim.

23. Dans une perspective plus générale, nous rappelons que l'analogie de l'horloge est un modèle philosophique classique, servant à prouver l'existence de Dieu, l'horloge étant la création et l'horloger le Créateur (Leibniz offre un développement connu de cette analogie). L'implication devient la suivante : dans leur orgueil, Sixte et Greslou tentent de remplacer Dieu. 
24. C'est justement dans le sens de «développement» que Bourget emploie le terme « évolution » ici - cet emploi en lui-même est révélateur. Il n'empêche que le mot est utilisé et la référence à la pensée spencérienne établie.

25. Patrick Tort, Spencer et l'évolutionnisme philosophique, Paris, PUF, 1996, p. 107-118, coll. «Que sais-je?».

26. On peut se demander si Bourget réussit, car le procédé manque de rigueur intellectuelle. Son personnage Greslou part d'un constat descriptif (la vie amoureuse est comme un combat) pour conclure à une norme prescriptive (il faut que je sois le plus fort); ce syllogisme fallacieux est connu sous le nom de sophisme naturaliste. On peut incriminer toute l'application de la théorie sixtienne par Greslou comme une erreur gigantesque : du fait que la vie psychique a des bases animales, on ne peut déduire que les hommes ont le droit de se conduire en bêtes. Or, c'est ce que Greslou conclut. Bourget voudrait nous faire croire que la démarche de son personnage est logique : l'application doit être correcte, si elle est censée démontrer les failles de la théorie incriminée.

27. Henri Klerkx souligne qu'à partir du Disciple Bourget évoluera « de l'étude psychologique à la morale »; Paul Bourget et ses idées littéraires, Nijmegen-Utrecht, Dekker \& Van de Vegt, 1946, p. 67.

28. Davidson a montré comment le « systematic statement " « of the character and doctrines » de Taine, entreprise dans les Essais de psychologie contemporaine, resurgit dans Le Disciple; « The Essais de Psychologie Contemporaine and the Character of Adrien Sixte», op. cit., p. 34 sq. Selon Davidson, Taine représente pour Bourget « the typical "modern" philosopher » (p. 48).

29. Sophie Spandonis, "Bourget, de Taine à Spencer, ou les paradoxes de "l'inconnaissable" ", Marie-Ange Fougère et Daniel Sangsue (dir.), Avez-vous lu Paul Bourget?, Dijon, Éditions Universitaires de Dijon, 2007, p.61-73, Dirk Hoeges, Literatur und Evolution. Studien zur französischen Literaturkritik im 19. Jahrhundert, Heidelberg, Carl Winter Universitätsverlag, 1980, p. 62 sq.

30. Lee souligne la référence à Kant, tout en présentant d'autres modèles, dont Taine, mais aussi Spinoza ; $c f$. «Bourget's Debt to Herbert Spencer : Le Disciple and the Self-Adjusting Watch », op. cit., p. 666 sq. Sur l'actualité (controversée) de Kant pour le monde intellectuel français de la fin $\mathrm{du} \mathrm{XIX}^{\mathrm{e}}$ siècle, $c f$. Jean-Louis Cabanès, "L'anti-kantisme dans les morales fin de siècle ", Romantisme, $\mathrm{n}^{\circ}$ 142, 4, 2008, p. 53-69.

31. Taine conclut sa lettre sur Le Disciple de la façon suivante: «Peut-être la voie que vous prenez, votre idée de l'inconnaissable, d'un au-delà, d'un noumène, vous conduira-t-elle vers un port mystique, vers une forme de christianisme. » Taine, Sa Vie et sa Correspondance, op. cit., t. IV, p. 293.

32. Ceci est vrai pour le Spencer, que Bourget connaît au moment de la conception du roman. Le philosophe anglais lui-même se heurtait à une ambiance intellectuelle plus sceptique dans les années 1880-1900, avec notamment des concepts tels la décadence et la dégénérescence: "Spencer lui-même ne resta pas totalement étranger à ce climat pessimiste, et se mit, dans les dernières années de sa vie, à envisager la possibilité d'une décadence sociale, tout en se refusant à admettre toute éventualité de dégénérescence biologique, dissociant ainsi l'unité de ses "lois universelles de la nature". » Daniel Becquemont, « Herbert Spencer : progrès et décadence ", Mil neuf cent, $n^{\circ} 14,1996$, p. 69-88, ici p. 81.

33. Cette définition est tirée de la dédicace à Hippolyte Taine; Paul Bourget, André Cornélis, CEuvres complètes, Paris, Plon, 1900, t. III : Romans I, p. 295.

34. Quant à cette dualité, la comparaison avec André Cornélis s'impose de nouveau : ce roman d'inspiration scientifique se présente comme une grande confession. Ibid., p. 297-299 et p. 488 sq.

35. Je développe ici l'idée esquissée chez Lee : «Even now, Bourget is clearly loath to renounce Taine's method entirely and, consistent with the strictures of De l'Intelligence, Le Disciple remains a work of pathology, offering no hero-norm of the values it promotes. " "Bourget's Debt to Herbert Spencer: Le Disciple and the Self-Adjusting Watch», op. cit., p.665. Il faut objecter, 
cependant, que le comte André représente justement une norme hérö̈que, qui gagne son lustre par le contraste avec Greslou et Sixte, personnages souffrant d'une crise et d'un discrédit moraux. Par ailleurs, Lee voit également une «satire» (p. 667) ou bien une «dimension parodique » (p. 668) à l'œuvre dans la présentation de Greslou; si la critique est sensible et peut prendre des formes comiques, notamment dans la présentation de Sixte, il nous semble exagéré d'aller aussi loin.

36. De l'Intelligence, op. cit., p. 3.

37. Lee, «Bourget's Debt to Herbert Spencer : Le Disciple and the Self-Adjusting Watch », op. cit., p. 668.

INDEX

Mots-clés : Bourget (Paul), positivisme, science, religion, Taine (Hippolyte)

\section{AUTEUR}

\section{NIKLAS BENDER}

Université de Tübingen 